\title{
A newly identified calculation discrepancy of the Sunset semi-continuous carbon analyzer
}

\section{G. J. Zheng et al.}

Correspondence to: K. B. He (hekb@tsinghua.edu.cn) and F. K. Duan (duanfk@mail.tsinghua.edu.cn) 

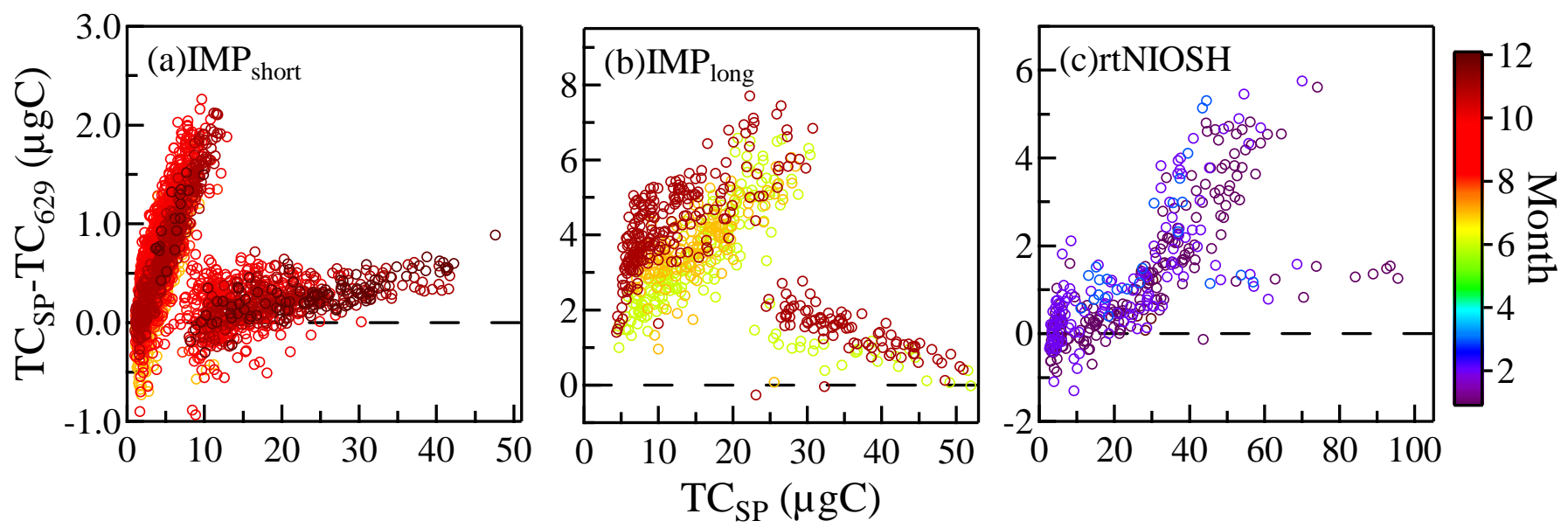

Figure S1. Comparison of TC results given by the newest version (629) of calculation software (TC629) and the single-point correction (TCsP), for ambient samples analyzed with (a) IMPshort, (b)IMPlong and (c) rtNIOSH protocol. 

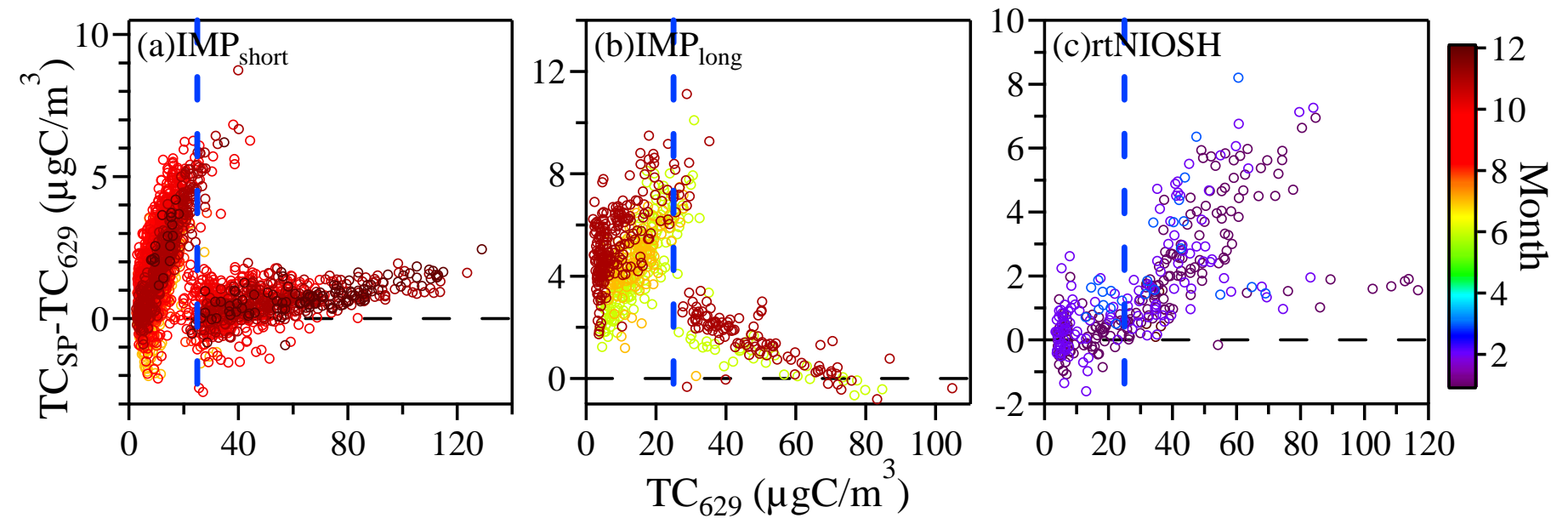

Figure S2. Comparison of TC concentration results given by the newest version (629) of calculation software (TC629) and the single-point correction (TCsP), for ambient samples analyzed with (a) IMPshort, (b)IMPlong and (c) rtNIOSH protocol 


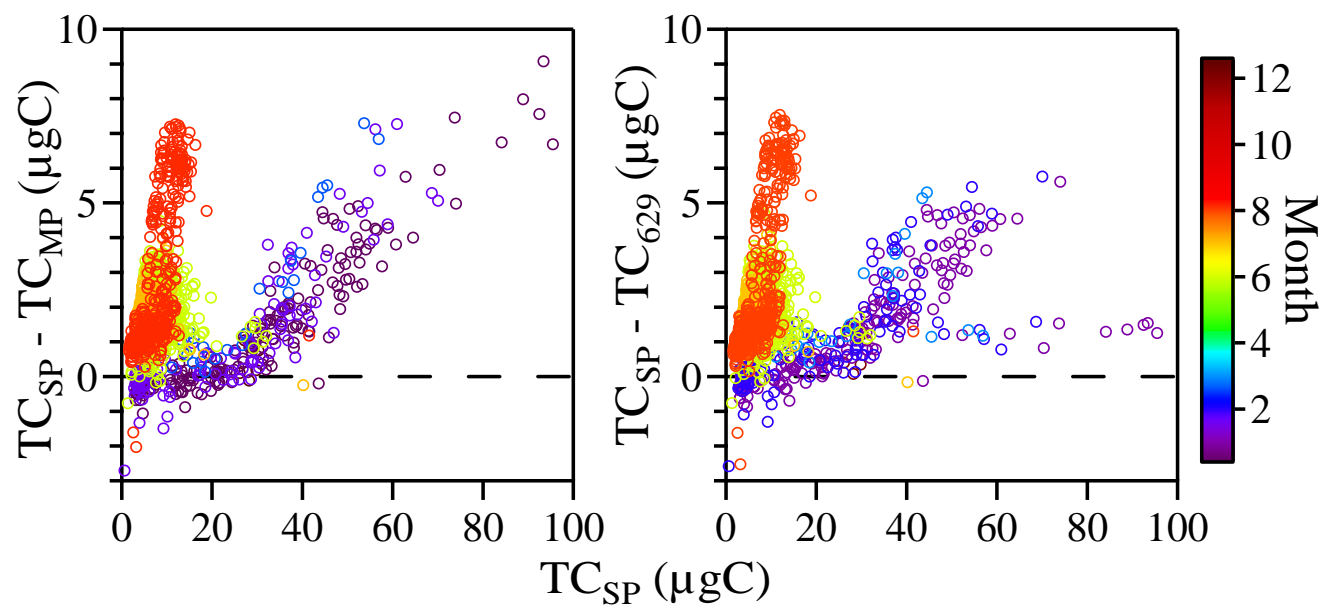

Figure S3. Seasonal variation of threshold carbon load for rtNIOSH protocol. Differences of TC concentration results given by the newest version (629) of calculation software (TC629), by the multi-point correction (TCMP) and by the single-point correction (TCsp) were compared. 